\title{
ION SOURCES FOR NANOFABRICATION AND HIGH RESOLUTION LITHOGRAPHY
}

\author{
J. Melngailis, ${ }^{*}$ University of Maryland, College Park, MD 20742-3511, USA
}

\section{Abstract}

Ion sources that are used to produce nanometer resolution patterns directly on the substrate are either point sources or volume plasma sources. The point sources are used in focused ion beam systems which focus an image of the point on the sample. These sources are, in practice gallium liquid metal. Other sources, such as gas field ion sources, are also being considered. Volume plasma sources are used with ion projection, where instead of the image of a point source, the image of a "back illuminated" stencil mask is projected on the substrate. The operation, application, and characteristics of the three types of sources are reviewed and compared.

\section{INTRODUCTION}

Nanofabrication can be regarded as consisting of material removal, addition, or alteration with nanometer resolution. It is also the key technology in the integrated circuits industry where gate lengths of transistors are now in the vicinity of $100 \mathrm{~nm}$. The semiconductor industry has been the main driving force in the development of the nanofabrication technology. In the semiconductor industry nanofabrication is based on lithography, i.e., exposure of a radiation sensitive polymer, called photoresist, with either photons or electrons which then renders it selectively soluble in an appropriate developer. The uncovered surface of the substrate is then partly etched away, implanted, or augmented. The patterned resist having served its purpose is removed and the process is repeated with a different pattern. In this way the multi-layer mosaic of transistors and conducting and insulating films which makes up our ubiquitous semiconductor chips are produced.

In the present-day semiconductor industry ion microbeams play a special role because they can be used in nanofabrication without this multi-step lithography process [1]. When a focused beam of ions is traced over a surface, it removes material to form a trench approximately equal to the beam diameter, which can be in the $10 \mathrm{~nm}$ range. In addition, if a precursor gas such as $W(C 0)_{6}$ is present and adsorbs on the surface, the ion beam breaks down the compound leaving the metal constituent behind. Thus focused ion beams can, and are, used to "rewire" integrated circuits by cutting or depositing metal film conductors. This is particularly useful in the pre-production development phase to alter a

*melng@glue.umd.edu circuit that may not be working because of a design or fabrication error. Otherwise, one would have to repeat the many steps needed to make a new circuit. Another way that focused ion beams are used is in defect diagnosis. If a contact is thought to be faulty, for example, a wide trench is milled in a circuit surface so that the edge of the trench intersects the faulty component. When the sample is tilted, the cross section of this component can be seen in a scanning electron microscope (or a scanning ion microscope, i.e. the same instrument that was used for cutting can, at a much dose, be used for viewing).

These focused ion beam processes are very local and slow and cannot be applied to manufacturing when the entire surface of a wafer is treated at once. Accordingly, other ways of producing a patterned dose of ions on a larger surface have been conceived. Three ways of producing ion nanobeams are illustrated in Fig. 1.

(a)

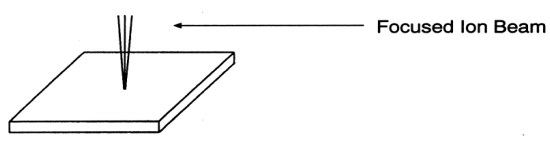

(b)

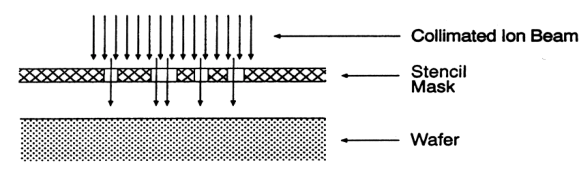

(c)

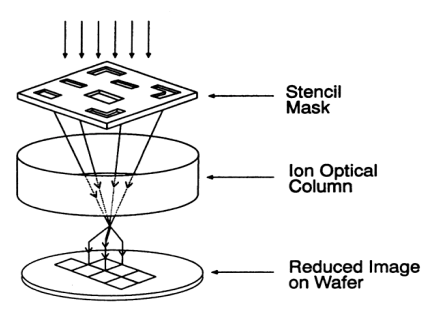

Fig. 1.Three ways of producing a patterned dose of ions on the surface: a) focused (point beams deflected in a programmed fashion, b) masked ion beam lithography, stencil mask in close proximity to wafewr, and c) stencil mask pattern projected (demagnified) on the substrate. 
The first in Fig. 1(a) is the focused ion beam that we have discussed up to now and that is used commercially. In this case a bright, point source of ions is focused to a point spot by means of suitable charged particle optics. The ions are accelerated to a high energy, usually in the $30-50 \mathrm{keV}$ range. The second way, Fig. $1 \mathrm{~b}$, is to have a broad, collimated beam pass through a stencil mask [2]. For experimental purposes, this technique does not represent a special challenge for ion sources since one can simply use an ion implanter and achieve collimation by having the ions pass through a long drift tube. The third method, Fig. 1c, called ion projection lithography [3], can be regarded as a combination of the other two. Here a collimated beam of ions is incident on a stencil mask, and the image of the stencil mask is projected demagnified onto the substrate. Although the source in this case presents some challenges, the main challenge in this technology is the ion optics, which must project an entire image with minimum distortion and simultaneously focus over the entire field. Contrary to the focused point beams, where the main applications are based on bypassing lithography, the ion image projection technique is mainly being developed for resist exposure with light ions such as helium or hydrogen, i.e. it represents a new type of lithography Although the source requirements for the three techniques are different, there are common elements.

\section{FIGURES OF MERIT OF ION SOURCES}

Figures of merit of ion sources [4].

\subsection{Brightness}

Particularly for the case of focused point beams, the ideal source would be a point that emits a high current of ions radially into a small solid angle. As in light optics a perfectly collimated beam would, of course, be equivalent. The unnormalized brightness is given by

$$
B=\frac{I}{\Omega A},
$$

where $I$ is the current, $A$ the area from which it is emitted, and $\Omega$ the solid angle into which the current is emitted. The units of brightness are $\left(A / \mathrm{cm}^{2} s r\right)$. Strictly speaking Eq. 1 only applies when the current into a given solid angle is constant. In general, Eq. 1 should be written in terms of differentials of the quantities, but it is useful as it stands for approximate calculations.

Since in most practical cases the ions are accelerated away from the source by some electric field, and since, for example, a uniform electric field over a large distance would produce a perfectly collimated beam of ions of infinite brightness, it is convenient to "normalize" and to define brightness as

$$
\beta=\frac{I}{\Omega A V},
$$

where $V$ is the potential through which the ions have been accelerated. This brightness is conserved in an ion optical system. Thus one can infer the brightness of the source by measuring the spot size, convergence angle, and beam voltage at the focal spot of an ion beam system where it is incident on the substrate. Particularly in the case of illumination of a stencil mask, one often speaks also of angular intensity

$$
B_{\Omega}=\frac{I}{\Omega} .
$$

\subsection{Energy Spread}

In the cases of interest the ions are focused from a point to a point (or from an image, such as in Fig. 1C, which can be regarded as a series of points to an, often demagnified, image). Ions of a different initial energy will have a different focal length when passing through an ion lens. Thus an initial energy spread of the ions emitted by a source will lead to a blurring of the image. (This is analogous to the chromatic blur in light optics, i.e., in a simple lens light of a different wavelengths has different focal lengths)

These are the main technical figures of merit. One can, of course, add stability, low cost, compact size, and ease of operation.

\section{SOURCES FOR FOCUSED ION BEAM (FIB) SYSTEMS}

Because FIB systems are practically useful, and because ion sources represent a special challenge, most of our attention will be focused on these sources.

\subsection{Liquid metal ion sources (MLIS)}

Liquid metal ion sources (LMIS) operating with Ga ions are most commonly used in FIB systems [5]. Figure 2 shows a typical $\mathrm{Ga}$ ion source with a coil acting as a reservoir and a sharpened tungsten needle from which the ions are extracted by an applied electric field. The coil and needle are spot welded to a tungsten wire loop which acts as a support and through which current can be passed to heat the metal and keep it molten. The molten metal slides down the needle and is pulled into a cusp (called a Taylor cone), by the applied electric field. The ions are emitted from the tip of this cusp, as illustrated schematically in Fig. 3. Remarkably this kind of source operating with $\mathrm{Ga}^{+}$ions is stable, bright, has an acceptable energy spread $(\Delta \mathrm{E} \sim 5 \mathrm{eV})$, and a long life ( 1000 hours). Sources with other metals, such as In, and with alloys such as $\mathrm{Au} / \mathrm{Si}, \mathrm{Au} / \mathrm{Si} / \mathrm{Bi}$, and $\mathrm{Pd} / \mathrm{As} / \mathrm{B}$ have also been 
developed but are more difficult to use and are not as stable or long-lived.

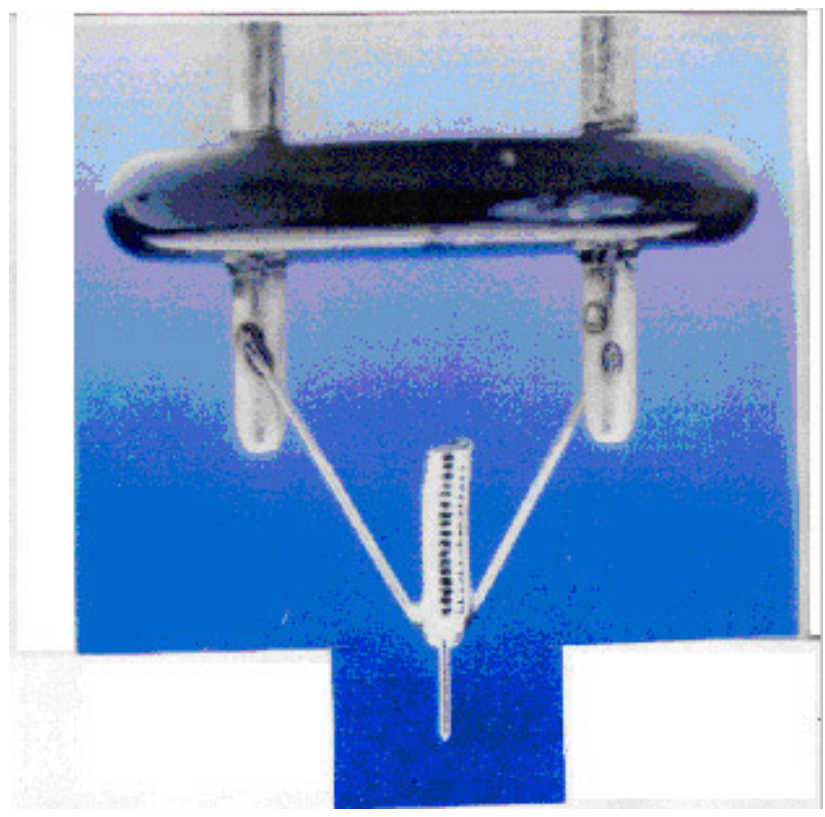

Fig. 2 Photograph of liquid metal ion source. The ceramic disk that holds the electrodes is a little less than $1 \mathrm{~cm}$. diameter.

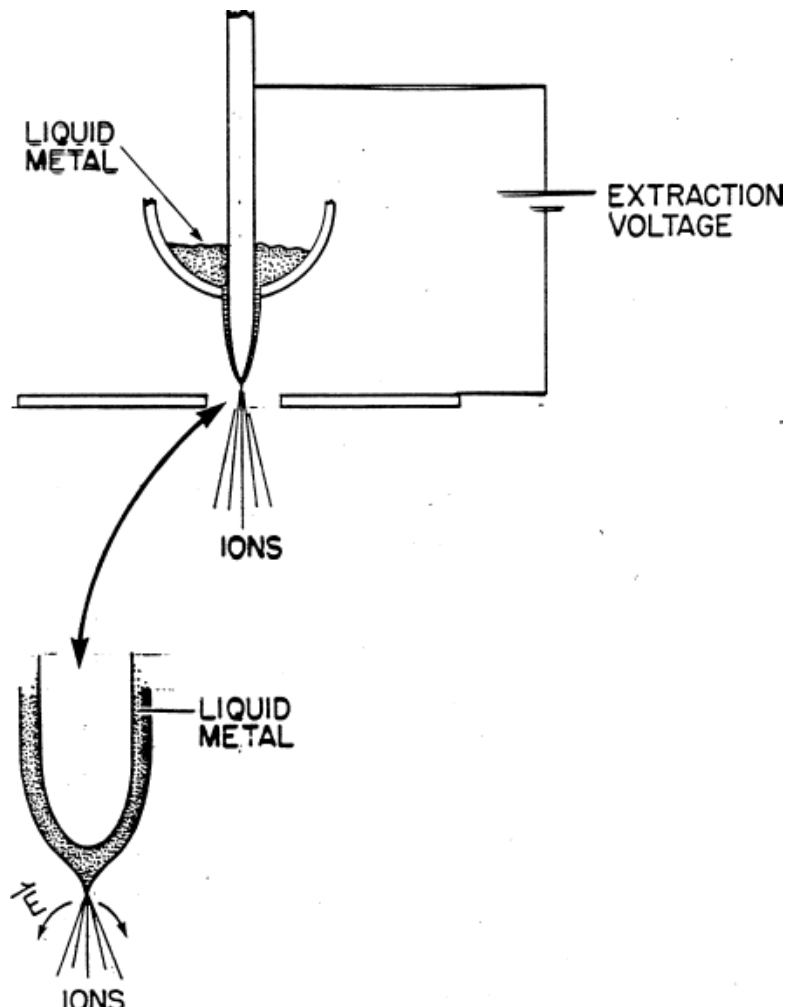

FIG. 3 Schematic of a liquid metal ion source. The electric field between the tip and a concentric aperture puls the liquid metal into a cusp from which the ions are emitted.

\subsection{Gas Field Ion Source}

The gas field ion source [6] has some geometric similarities with the liquid metal ion source except that the needle is cooled to cryogenic temperatures and gaseous species such as $\mathrm{H}, \mathrm{He}, \mathrm{Ne}, \mathrm{Ar} \ldots$ are condensed on the needle, as shown schematically in Fig. 4. Again the ions are extracted by applying a voltage between the

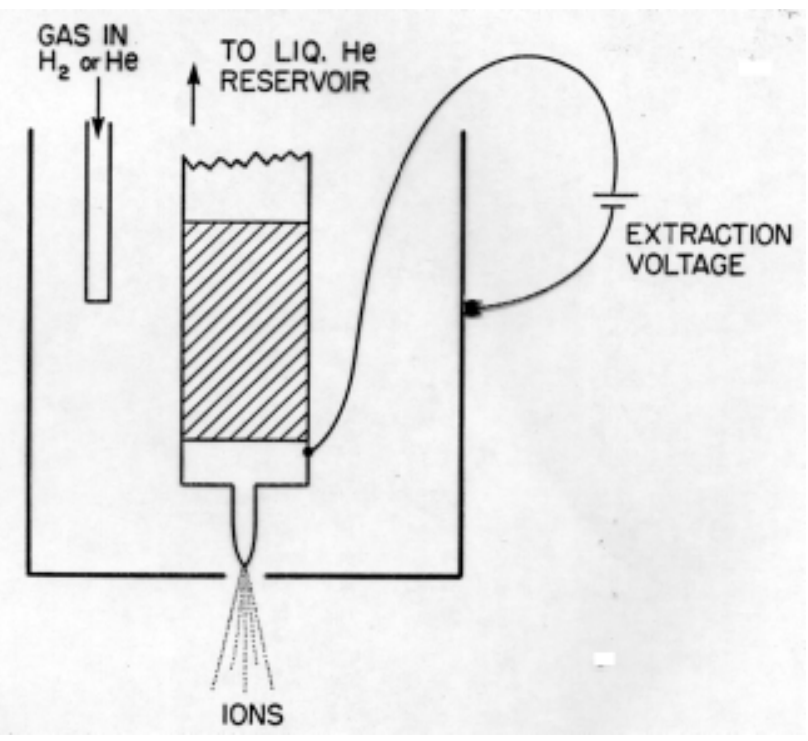

Fig.4 Schematic of the gas field ion source. If the tip from which the extraction occurs has a "super tip" the ion emission is confined to a narrow $\left(\sim 0.5^{0}\right)$ cone.

tip and a concentric electrode below the tip. With a sharpened tungsten needle tip of a few microns radius the ion source characteristics are similar to those of the liquid metal source. However, by special techniques it is possible to grow a "super-tip", a protrusion of a few nanometers at the apex of the tip. Because the emission current density is exponentially dependent of the electric field strength, the small increase in electric field at this protrusion causes a large increase in current. A few nanometers away from the protrusion the electric field lines seen by the ions appear parallel (given the relatively "large" tip radius). Thus the ions are immediately accelerated into a narrow, almost collimated beam. This leads to an ion source of extraordinary brightness. Unfortunately, although 160 hours of stable operation has been reported [7], these sources are in general difficult to operate and have not been incorporated into commercial systems.

\subsection{Volume plasma sources}

Volume plasma sources [8] in various configurations are the most common and are used in ion milling machines, ion implanters, and other ion accelerators. In these sources the desired species is introduced as a gas, and electron bombardment is used to ionize it. Ions are then extracted by suitable electrodes. Clearly, because the 
emission is not concentrated at a point as it is in the other two sources, the volume plasma sources are much less bright.[9] However, they emit larger currents, have high angular intensity, and are used as sources for masked ion beam lithography (Fig. 1b) and for ion projection lithography (Fig. 1c).

\section{DISCUSSION}

The properties of the ion sources are compared in Table I. Note that the plasma sources are more than three orders of magnitude less bright than the liquid metal. Thus the current in a given focal spot of a FIB system would be reduced by the same factor, and most practical applications would take prohibitively long times. Because of the high angular intensity, however, the plasma sources are widely used in implanters and in the ion projection systems being developed (Fig. 1c).

The main challenge in ion sources for focused beam systems is to find sources of ions that work as well or beter than the $\mathrm{Ga}^{+}$liquid metal source and that emit other ion species. In many applications $\mathrm{Ga}^{+}$ions act as a contaiminant. In the repair of photmasks milling off the chrome layer implants and stains the underlying quarts and reduces its optical transmission. Ga is a dopant of silicon and so any milling operation locally contaminates the sample. Noble gas ion sources would be very useful. So would many other species. Oxygen or cesium for high resolution scanning ion mass spectrometry (SIMS), arsenic, phosphorous, or boron for local, point-by-point controlled doping of silicon, berylium or silicon ions for doping GaAs, erbium ions for doping optical structures, etc. The gas field ion sources and the liquid metal ion source are unique in that the very high electric field at the sharp tip (in some cases sharp on an atomic scale) generates an ion current with very high density, and surprisingly, not very high energy spread. This condition is hard to duplicate in a volume plasma because of the particle repulsion and because of their random motion (i.e., plasma temperature). In fact, even in the liquid metal ion source the initial source size is determined by the ion-ion repulsion in the region near the tip where the density is high and the ion velocity is still low [10]. Similarly the ion-ion repulsion in the direction of ion travel is the main contributor to $\Delta E$. Accordingly, $\Delta E$ increases as the total current extracted from the source is increased

Table I. Comparison of various ion sources. Unnormalized brightness is most often quoted in the literature. For most sources an extraction voltage near $10 \mathrm{kV}$ is used, and for FIB systems a central few milliradian part of the beam is selected by a beam defining aperture. The brightness quoted divided by $10 \mathrm{kV}$ then would be the normalized brightness which is conserved as the beam passes through the rest of the column. For example, in ref 9 the image side brightness at $30 \mathrm{kV}$ is quoted as $1.65 \times 10^{3}$ which is equivalent to a source side brightness at $10 \mathrm{kV}$ of $0.55 \times 10^{3}$.

\begin{tabular}{|l|c|c|c|c|c|}
\hline $\begin{array}{l}\text { Type of ion } \\
\text { source }\end{array}$ & Ion Species & $\begin{array}{c}\text { Virtual source } \\
\text { size } \\
(\mathrm{nm})\end{array}$ & $\begin{array}{l}\text { Energy } \\
\text { spread, } \Delta \mathrm{E} \\
(\mathrm{eV})\end{array}$ & $\begin{array}{l}\text { Unnormalized } \\
\text { brightness, B } \\
\left(\mathrm{A} / \mathrm{cm}^{2} \mathrm{sr}\right)\end{array}$ & $\begin{array}{c}\text { Angular } \\
\text { brightness } \\
(\mu \mathrm{A} / \mathrm{sr})\end{array}$ \\
\hline Liquid metal & $\mathrm{Ga}^{+}$ & 50 & $>4$ & $3 \times 10^{6}$ & 50 \\
\hline $\begin{array}{c}\text { Gas field ion } \\
\text { (supertip) } \\
\text { (ref. 11) }\end{array}$ & $\mathrm{H}^{+}, \mathrm{H}_{2}{ }^{+}, \mathrm{He}^{+}$ & 0.5 & $\sim 1$ & $5 \times 10^{9}$ & 35 \\
\hline $\begin{array}{c}\text { Multicusp } \\
\text { Plasma } \\
\text { (ref.9) }\end{array}$ & $\mathrm{Kr}^{+}$ & 17 & $1-3$ & $0.55 \times 10^{3}$ & 40 \\
\hline $\begin{array}{c}\text { Penning } \\
\text { (pulsed) } \\
\text { (ref. 12) }\end{array}$ & $\mathrm{Ar}^{+}$ & 4.5 & $10^{3}$ & \\
\hline
\end{tabular}




\section{REFERENCES:}

[1] There are a number of reviews of FIB technology. For example. M. Utlaut, Ch. 11 "Focused ion beams" in Handbook of Charged particlreoptics, J. Orloff, Editor. CRC Press (1997), R. Levi-Setti, "High resolution scanning transmission low energy ion microscopes and microanalyzers" Adv. In electronics and electron physics, Suppl. 13A p. 261, (Academic Press 1980), J. Melngailis, "FIB technology and applications" (Review paper) J. Vac. Sci. Technol. B5, 469-495, (1987)

[2] J. Randall, J.Vac.Sci. Technol. A4, 777 (1986), J. L Bartelt, Solid State Technology., Vol29, 215 (May 1986), M.D. Morgan, W.E. Horne, V. Sundaram, J.C.Wolfe, S. Pendharkan, and R.Tiberio, J. Vac. Sci. Technol. B14,3903, (1996)

[3] H. Loeschner, , G. Stengl, R. Kaesmaier, and A. Wolter, J.Vac. Sci. Technol. B19, (Nov/Dec. 2001) to be published. J. Melngailis, A.A. Mondelli, I. L.Berry and R. Mohondro, J. Vac. Sci. Technol B16, 927-957 (1998)
[4] R. Levi-Setti (Ref. 1)

[5] P.D. Prewett, G.L.R.Mair Focused Ion Beams from Liquid Metal Ion Sources Research Studies Press/ J.Wiley\&Sons (1991) Also G.R.L. Mair in Ref. 1 Handbook.. J. Orloff. Ed.

[6] Ch. Wilbertz, et.al. Nucl. Instr. and Methods in Physics ResearchB63, 120, (1992)

[7] R Boerret, K. Jousten, K. Boehringer, and S. Kalbitzer, J. Phys. D, Appl . Phys. 21, p. 1835 (1988)

[8] K.N. Leung, et. al. J.Vac Sci. Technol. B13, 2600 (1995)

[9\} L. Scipioni, et. al. J.Vac Sci. Technol B18, 3194 (2000)

[10] J.W. Ward, . J.Vac Sci. Technol. B3, 207, (1985) 